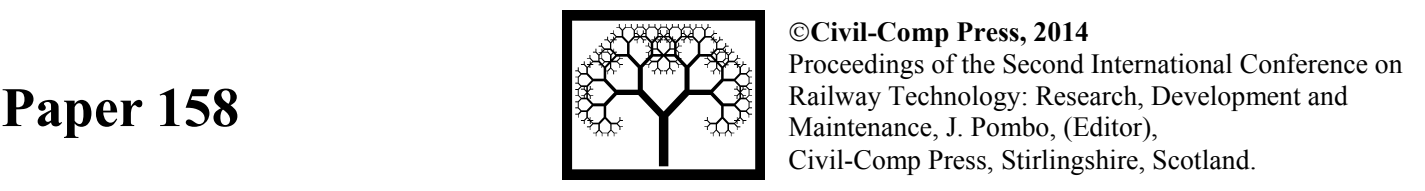

\title{
Comparison of Braking Performance for Different Technologies of Heavy Hauled Freight Trains
}

\author{
L. Cantone and V. Vullo \\ Department of Engineering for Enterprise "Mario Lucertini" \\ University of Rome "Tor Vergata", Rome, Italy
}

\begin{abstract}
The paper shows a comparison of different braking technologies for freight trains and their effects on in-train forces. At this aim, the UIC certified software TrainDy is employed. Freight train sets, having one and two remote locomotives, are investigated both as coupled trains and as fixed train compositions. There is also an evaluation of the in-train forces of freight train sets equipped by electro-pneumatic brakes and disk brakes. The case of two remote locomotives is further explored showing the minor benefits of an ad hoc activation of drivers brake valve, compared to the benefits of an optimized mass distribution, for train sets both coupled and fixed.
\end{abstract}

Keywords: longitudinal dynamics, freight train, interoperability.

\section{Introduction}

Whilst on the AAR (Association of American Railroads) market the freight train already evolved embarking electronics for smarter power and braking control, the European railway freight transportation still needs an increment of productivity in many Countries. There are several national and international projects (also financed by European Commission) aiming to enhance such transportation type. General aim of such projects is focused on raising competitiveness of railway freight transportation, which is most suitable especially for long distances, i.e. for interoperable freight traffic. For economic reasons, Railway Undertakings aim to obtain such benefits by using conventional freight wagons (also revamped) and/or by buying new locos or by simply upgrading old ones. Of course, this approach needs the implementation of new ideas and concepts, i.e. new operational rules, distributed traction/braking and so on.

One of the main obstacles to overcome, in order to really obtain a growth of efficiency, is reducing the derailment and disruption risk of such new and more 
productive trains. Wagon derailment strongly depends on the wheel/rail contact conditions; among several types of derailment mechanisms [1] there is a particular one that is connected to the high in-train compressive forces, especially when they occur on short radius curves. Limiting such in-train (or longitudinal) forces results in reducing the derailment risk and the disruption risk of trains. Longitudinal Compressive Forces (LCF) and Longitudinal Tension Forces (LTF) are mainly caused by the features of the standard UIC braking system, which equips the majority of conventional freight wagons and that does not allow synchronous braking forces among wagons, resulting in (potentially) high LCF and LTF.

In the past years, at the University of Rome Tor Vergata, new research software, called TrainDy, has been developed by means of the industrial and economic support of Faiveley Transport of Italy. Such software has been subsequently certified by UIC [2]-[5] for the computation of in-train forces for freight trains also for train make ups with distributed traction/braking; the validation of this software has been carried out by comparison of the numerical results with the measurements of more than 30 experimental tests made available by DB AG, SNCF and Trenitalia. This paper starts by stating a number of possible scenarios for heavy hauled freight trains, whose mass is in agreement with the UIC 421 [6] for the interoperability of freight trains, and it investigates the effect of payload distribution along the train on longitudinal in-train forces. Then new types of freight trains are investigated, equipped by electro-pneumatic brake and loco placed at the beginning of the train. Finally, the paper analyses freight trains equipped with one or two remote locos and in the latter case an investigation on the effect of an asynchronous activation of braking is performed, showing that braking performances can be improved by means of an ad hoc activation sequence of brake pipe venting.

The assembling of trains is made by an automatic routine that generates trains randomly according to a prescribed value of mass/length and other parameters, as it will be shown in the next section.

\section{Generator of random trains}

In this section, a numerical algorithm, already described in [7], is recalled for easiness of reading. Such algorithm is based on an algebraic model and it is alternative to the (optional) algorithm reported in Appendix B of leaflet UIC 421 for the automatic generation of freight train sets. The created freight train sets respect the requirements in terms of length $\left(L_{T}\right)$ and mass $\left(M_{T}\right)$; moreover, definition of train sets can allow the specification of: fraction $\left(F_{i}\right)$ of each vehicle model; ii) for each vehicle type, the fraction $\left(f_{i j}\right)$ of hauled mass $\left(m_{i j}\right)$. The previous variables, $L_{T}, M_{T}$, $F_{i}, f_{i j}, m_{i j}$, are the input for the algebraic solver along with the model of vehicles in freight train sets: the number of different wagon models is $n_{v}$; former inputs allow the computation of the number of vehicles of a specific model having a prescribed mass, indicated by $n_{i j}$; such quantities, in turn, permit the assembling of train sets. The number of different hauled masses carried by the wagon $i$ is indicated by $n_{m i}$; the total number of unknowns $n_{i j}$ is equal to $\sum_{i=1}^{n_{v}} n_{m i}$. 
Hence, $n_{i j}$ is the number of wagons of model $i$ having the mass $j$ (previously indicated by $m_{i j}$ ), the wagons of model $i$ in the current train set are:

$$
n_{i}=\sum_{j=1}^{n_{m i}} n_{i j}
$$

The number of wagons of current train set is $n_{T}=\sum_{i=1}^{n_{v}} n_{i}$.For the fractions of wagon model of the train set it is possible to write: $F_{i}=\frac{n_{i}}{\sum n_{i}}$. By explicating former relationship and using eq. (1) for different wagon models, it is possible to write $\left(n_{v^{-}}\right.$ 1) relationships such as:

$$
F_{i} \sum_{j=1}^{n_{m 1}} n_{1 j}+F_{i} \sum_{j=1}^{n_{m 2}} n_{2 j}+\cdots+F_{i} \sum_{j=1}^{n_{m m_{v}}} n_{n_{v j}}=\sum_{j=1}^{n_{m i}} n_{i j}
$$

For the fractions of hauled masses carried by the $i^{\text {th }}$ wagon, it is possible to similarly write: $f_{i j}=\frac{n_{i j}}{n_{i}}$. Also in this case, by using eq. (1), it is possible to write, for each wagon model, $\left(n_{m i}-1\right)$ relationships such as:

$$
f_{i j} \cdot n_{i 1}+f_{i j} \cdot n_{i 2}+\cdots+f_{i j} \cdot n_{i n_{m i}}=n_{i j}
$$

Wagon length is indicated by $l_{i}$; this means that train length is:

$$
l_{1} \sum_{j=1}^{n_{m 1}} n_{1 j}+l_{2} \sum_{j=1}^{n_{m 2}} n_{2 j}+\cdots+l_{n_{v}} \sum_{j=1}^{n_{m n_{v}}} n_{n_{v} j}=L_{T}
$$

Mass train is equal to:

$$
\sum_{i, j} n_{i j} \cdot m_{i j}=M_{T}
$$

If the inputs contain both mass and length of the train the problem is overdetermined: there is an equation more than the number of unknowns. In spite of this, it is possible to automatically generate train sets respecting the mass and length inputs by violating the fractions of wagon model or the fractions of mass. Moreover, since the solution is given by integer numbers, even if only the mass or the length is prescribed, the solution is computed by inverting the matrix of order $\sum_{i=1}^{n_{v}} n_{m i}$ and then by minimising the error in order to obtain the final solution.

\section{Input data}

Table 1 reports the main data of wagons used in the simulations: they are all container-type wagons. In the automatically assembled freight train sets, such wagons are employed with the same percentage. Table 2 reports the hauled mass 
values for each vehicle (formerly indicated by $m_{i j}$ ) along with the associated fraction (formerly indicated by $f_{i j}$ ). By using former symbols it can be stated that coefficients $F_{i}$ are equal to $1 / 6, n_{v}$ is equal to 6 and coefficients $n_{m i}$ are all equal to 2 . Furthermore, $f_{11}$ is equal to $0.25, f_{12}$ is equal to $0.75 ; m_{11}$ is equal to $70, m_{12}$ is equal to 135 and so on.

\begin{tabular}{|c|c|c|c|c|c|c|}
\hline & Sggmrs715 & Sgmmns-738 & Sgns691 & Sgns692 & Sgns705 & Sgns 735 \\
\hline Length $[\mathrm{m}]$ & 33.94 & 13.13 & 19.74 & 19.64 & 19.74 & 19.64 \\
\hline Tare $[t]$ & 30 & 19.38 & \multicolumn{4}{|c|}{20} \\
\hline Axes number & 6 & \multicolumn{5}{|c|}{4} \\
\hline$\%$ usage & \multicolumn{6}{|c|}{$1 / 6$} \\
\hline Braking device & \multicolumn{6}{|c|}{ Block brake, iron shoes (also disk brake for EP brake) } \\
\hline $\begin{array}{l}\text { Braking regime } \\
{[8]-[9]} \\
\end{array}$ & \multicolumn{6}{|c|}{$\begin{array}{l}\mathrm{G} \text { (typical filling times of braking cylinders } 24 \mathrm{~s} \text { and } 28 \mathrm{~s} \text { ) } \\
\mathrm{P} \text { (typical filling times of braking cylinders } 4 \mathrm{~s} \text { and } 4.5 \mathrm{~s})\end{array}$} \\
\hline \multicolumn{7}{|c|}{ Table 1: Main wagon data } \\
\hline & Sggmrs715 & Sgmmns-738 & $\begin{array}{c}\text { Sgns69 } \\
1\end{array}$ & $\begin{array}{c}\text { Sgns69 } \\
2\end{array}$ & $\begin{array}{l}\text { Sgns70 } \\
5\end{array}$ & $\begin{array}{c}\text { Sgns73 } \\
5\end{array}$ \\
\hline \multirow{2}{*}{$\begin{array}{c}\text { Gross mass [t] / } \\
\text { Usage }[\%]\end{array}$} & $70 / 25$ & $55 / 25$ & $50 / 20$ & $60 / 20$ & $55 / 20$ & $20 / 30$ \\
\hline & $135 / 75$ & $90 / 75$ & $90 / 80$ & $90 / 80$ & $90 / 80$ & $90 / 70$ \\
\hline
\end{tabular}

Table 2: Mass distribution for each wagon

It is worthwhile mention that data used in Table 2 are only an example and they do not represent any existing case, even if the data are reasonable; data of Table 1 are taken by the database of TrainDy. In spite of this, following results are of interest for the assessment of freight train sets.

Freight train sets investigated here after start from actual admissible gross mass/braking regime couples and explore the braking performances (mainly respect to in-train forces and stopping distances) of heavier and longer trains, equipped by not common braking systems (for freight trains), i.e. electro-pneumatic brake, brake pipe accelerators valves and remote locos.

\section{Loco at the beginning of freight train set}

Actual high performance freight trains (in terms of gross mass), typically running in Europe, employ Long Locomotive (LL) braking regime (hauled mass $1600 \mathrm{t}$ ) or Goods $(\mathrm{G})$ braking regime (hauled mass of $2500 \mathrm{t}$ ). Heavier trains circulate but they are subjected to specific rules. Figure 1 reports the cumulative function of in-train 
forces (negative sign means a compressive force caused by approaching wagons and positive sign means a tensile force caused by moving wagons away). Figure legend reports also the type of performed train operation: E means emergency braking from $30 \mathrm{~km} / \mathrm{h}$ and TE means acceleration from zero speed up to $30 \mathrm{~km} / \mathrm{h}$ and then emergency braking, the latter is, as well known, the most severe for in-train forces. Compressive in-train forces are here reported by means of $10 \mathrm{~m}$ longitudinal compressive force $\left(\mathrm{LCF}_{10 \mathrm{~m}}\right.$ i.e. the minimum absolute value of the compressive force obtained in the previous $10 \mathrm{~m}$ ), whereas the tensile in-train forces are reported in terms of $2 \mathrm{~m}$ longitudinal tensile force $\left(\mathrm{LTF}_{2 \mathrm{~m}}\right.$, defined as the longitudinal compressive force, but referred to tensile force and $2 \mathrm{~m}$ ). This choice has been done since derailment due to excessive in-train compressive force is governed, according to railway experience, by $\mathrm{LCF}_{10 \mathrm{~m}}$ whereas train disruption is caused by almost instantaneous values of tensile forces at draw gears.

The figure shows that LL braking regime cause severe tensile forces and that, also during an emergency braking, $\mathrm{LCF}_{10 \mathrm{~m}}$ for $\mathrm{G}$ regime can overcome the typical admissible value of $400 \mathrm{kN}$ of high performance wagons. Values of in-train forces reported in Figure 1 shall be used as reference for other cases shown hereafter.

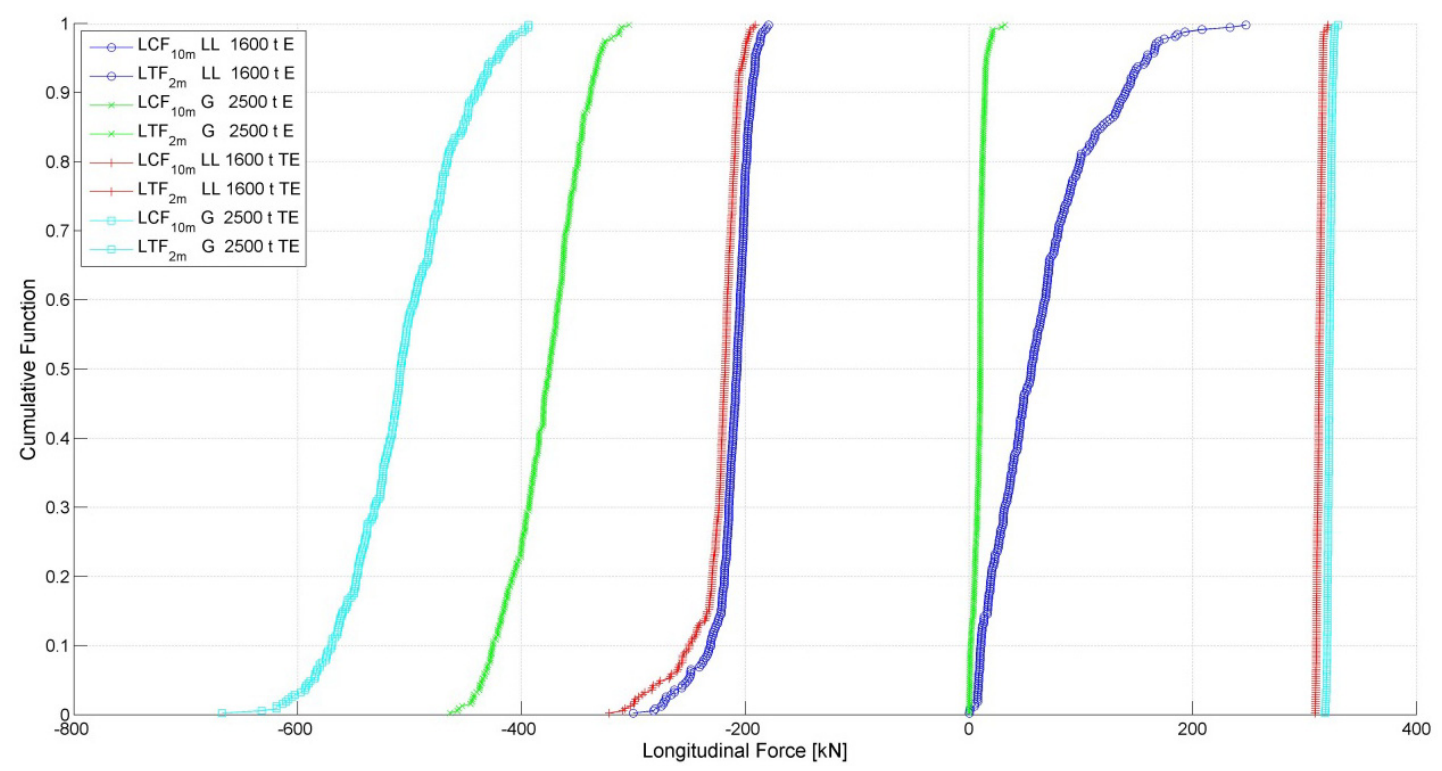

Figure 1: Cumulative function for traditional trains performing different train operations.

Another train set concept with loco placed at the beginning of the train is the one that employs electro-pneumatic brakes. As an example, Figure 2 shows the cumulative function for freight train sets with wagon characteristics as in section 3 but equipped by electro-pneumatic braking and disk brakes. Train sets have a nominal length of $1500 \mathrm{~m}$ and brake according to passenger regime; as previously, starting speed for emergency braking is nominally $30 \mathrm{~km} / \mathrm{h}$. Results here reported show that there is a significant reduction of in-train forces both compressive and tensile: synchronous discharging of brake pipe and disk brake equipment guarantee such low value for in-train forces also in P regime, allowing a very high train mass. 


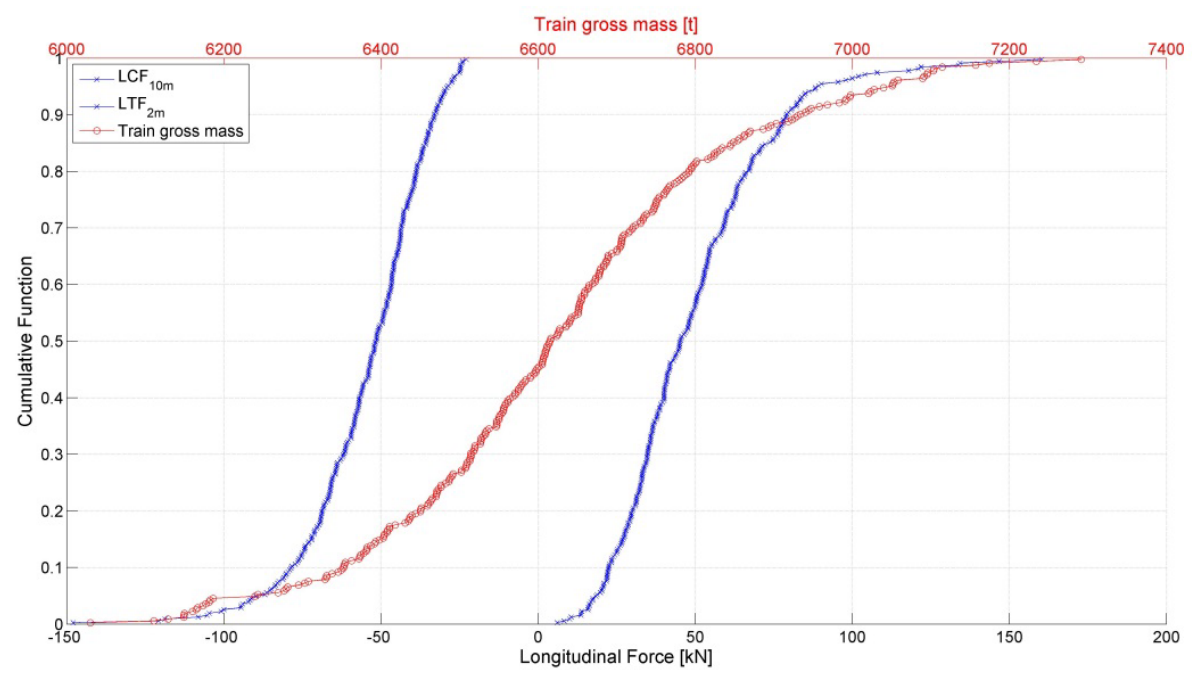

Figure 2: Cumulative function for EP brake.

\section{One remote loco}

This section shows an extensive investigation on the braking performance of freight trains equipped with a remote locomotive. Firstly, performance of two equal trains coupled together are investigated: the interval of investigation of single train is 500$850 \mathrm{~m}$ (excluded loco), i.e from a typical long Italian train up to an innovative specific train approved by DB AG; the investigated hauled mass of the single train is in the interval in 2000-2600 t.

(a)
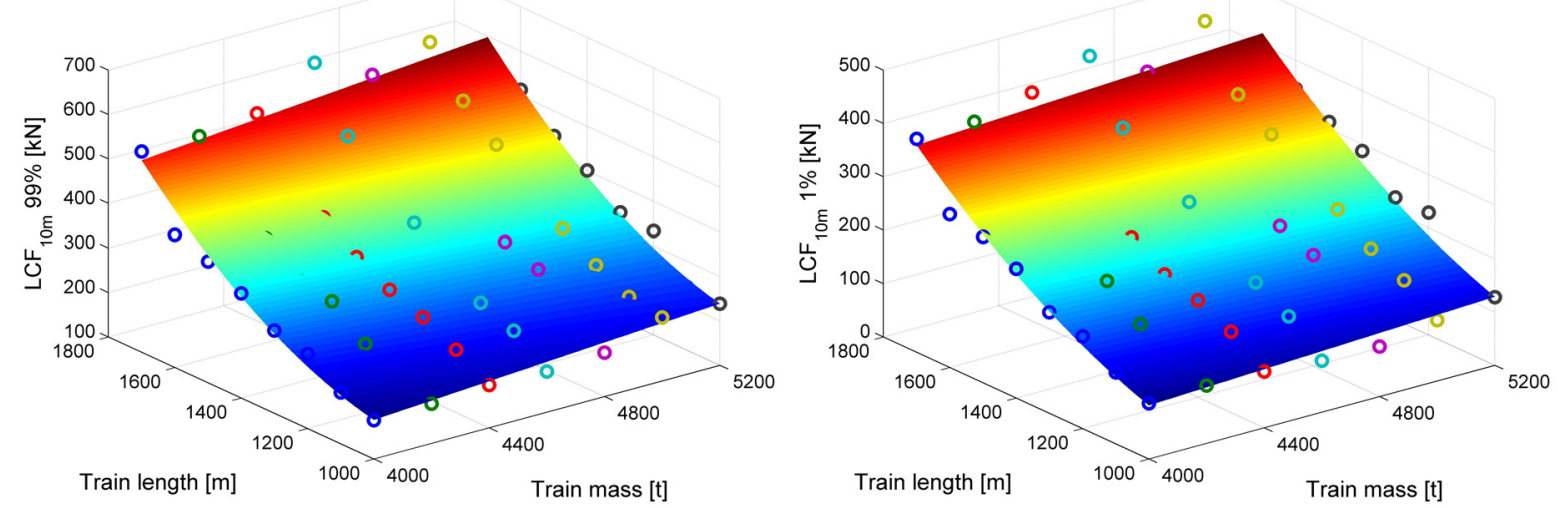

Figure 3: (a), $\mathrm{LCF}_{10 \mathrm{~m}} 99 \%$ percentile; (b), $\mathrm{LCF}_{10 \mathrm{~m}} 1 \%$ percentile.

Figure 3 reports the $10 \mathrm{~m}$ longitudinal compressive forces $\left(\mathrm{LCF}_{10}\right)$, with positive sign, for different length and mass of the overall train. Figure 3 (a) reports, for each train, value of $99 \%$ percentile (only $1 \%$ of trains have $\mathrm{LCF}_{10 \mathrm{~m}}$ bigger than indicated value), whereas (b) refers to $1 \%$ percentile (only $1 \%$ of trains have a $\mathrm{LCF}_{10}$ smaller then indicated values). Values in graph (a) provide information on achievable forces 
when freight train sets are assembled randomly, whereas values in graph (b) indicate the best attainable performance when train sets are optimally assembled. It is clear that, by optimizing the mass distribution along the train, it is possible to run heavy train sets with (comparably) low in-train forces. Figure 3 also reports, for each percentile, a polynomial fitting surface that interpolates data, represented by circles; moreover, such figure clearly shows that $\mathrm{LCF}_{10 \mathrm{~m}}$ increase more with train length than with train mass.

Figure 4 shows the contour plots associated to the fitting surfaces of Figure 3, in order to more easily evaluate the maximum gross weight of the train, fixing a value for train length and for admissible LCF. It is worthwhile to mention that having a high value of $\mathrm{LCF}_{10 \mathrm{~m}}$ does not automatically mean that train will derail; this usually occurs if such high value is undergone on a short radius curve and between wagons having a different load, so that the height of buffer heads are different. In turn, this means that in order to properly establish the derailment risk it is necessary a more complete 3D analysis of the forces exchanged by consecutive wagons, by means of their buffers [10]; nevertheless such studies of longitudinal dynamics are more than enough for a first evaluation phase. Figure 4 emphasizes what already told before: i.e. expecting an admissible $\mathrm{LCF}_{10}$ of $400 \mathrm{kN}$, mass optimization allow the composition of train sets whose length is $1600 \mathrm{~m}$ and whose mass is $5000 \mathrm{t}$. Of course, what here stated assumes that brake pipe discharging is synchronous between loco, otherwise further studies have to be accomplished in order assure train safety.
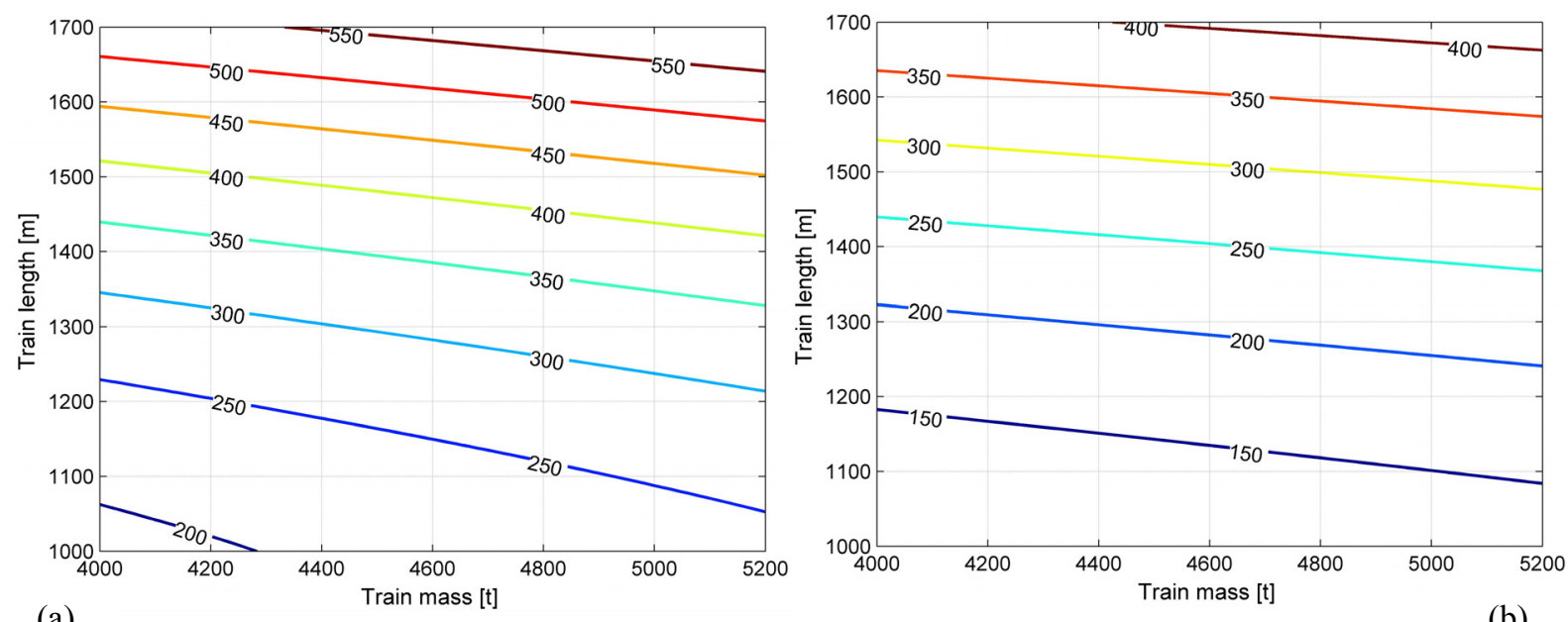

(a)

Figure 4: Contour plots for $\mathrm{LCF}_{10}$ : (a) 99\% percentile; (b) $1 \%$ percentile.

In order to provide a more precise evaluation of in-train compressive forces variability, Figure 5 displays the standard deviation for the train sets investigated so far: (a) shows the punctual values along with the polynomial fitting surface; (b) reports the contour plots corresponding to the fitting surface. Such figure clearly show that dispersion of in-train forces increases both with train length and with train mass. 


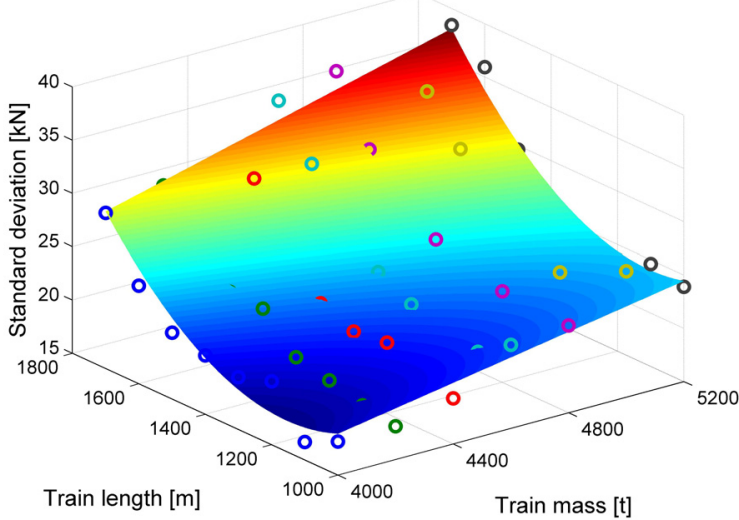

(a)

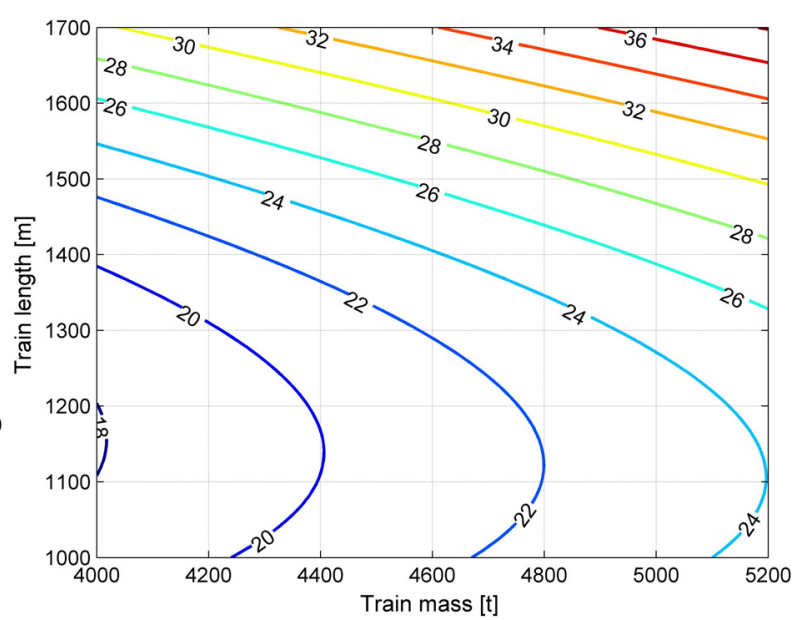

(b)

Figure 5: (a) standard deviation punctual and fitted; (b) corresponding contour plots

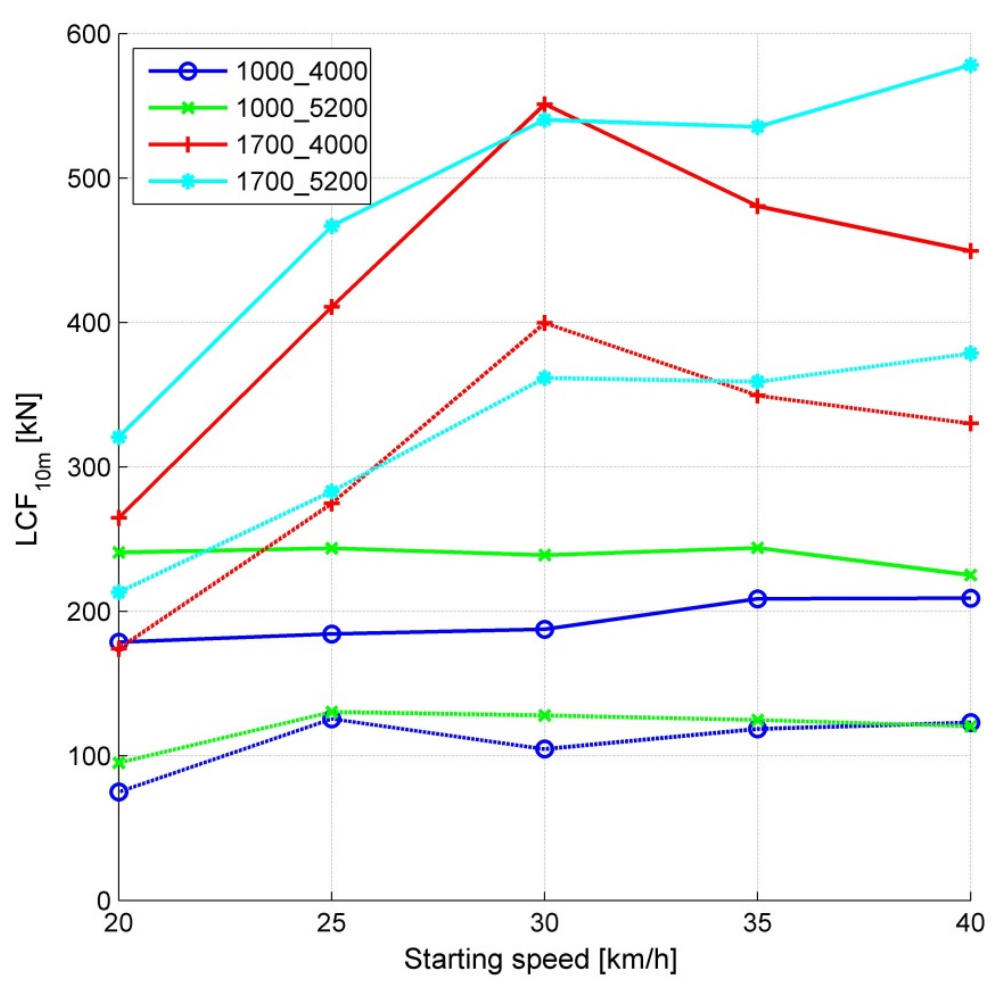

Figure 6: $\mathrm{LCF}_{10}$ evolution with starting speed: for each train set, $99 \%$ percentile is in solid line and $1 \%$ percentile is in dashed line.

Results reported so far refer to synchronous emergency braking from $30 \mathrm{~km} / \mathrm{h}$; Figure 6 clarifies the dependency of $\mathrm{LCF}_{10}$ by the starting speed (speeds from 20 $\mathrm{km} / \mathrm{h}$ up to $40 \mathrm{~km} / \mathrm{h}$ step $5 \mathrm{~km} / \mathrm{h}$ are here investigated): values referred to $99 \%$ percentile are displayed by a solid line, values referred to $1 \%$ percentile are displayed by a dashed line. The curves reported in Figure 6 refer to the four extreme 
combinations of mass/length intervals, formerly considered: increasing of mass/length of train sets results in a bigger dependency of $\mathrm{LCF}_{10}$ with the starting speed; as a consequence, a complete study of heavier and longer trains requires an examination of different starting speeds.

Up to now, the two sub-train of the whole train set had same length and mass, even if mass distribution was different (as it happens in the reality); as a consequence remote loco was always placed in the middle of the train. Figure 7 refers to a train with a hauled mass of $5000 \mathrm{t}$ and it investigates the effect of a different position of remote loco on in-train forces; as previously stated, solid lines refer to $99 \%$ percentile, whereas dashed lines refer to $1 \%$ percentile; label ' $800 \_600$ ' indicates that the length of the first sub-train is $800 \mathrm{~m}$ and that the length of second sub-train is $600 \mathrm{~m}$ (similar consideration applies for other labels); (a) refers to $\mathrm{LCF}_{10 \mathrm{~m}}$ whereas $(\mathrm{b})$ refers to $\mathrm{LTF}_{2 \mathrm{~m}}$. Figure 7 clearly shows that placing the remote loco towards the end of the train causes a reduction of in-train compressive forces and an increase of in-train tension forces, mainly acting on the first sub-train, since remote loco vents the brake pipe of both trains. It is worthwhile to mention that the chosen train doubles the mass and the length of the heavier and longer train, admitted by the UIC 421. Furthermore, such results allow asserting that better results in terms of in-train forces can be achieved by means of fixed train compositions, instead of compositions with coupled trains; according to showed results the best placement of remote locomotive is around $2 / 3$ of the train; such positioning guarantees also a reduced dependency of in-train forces with starting speed.

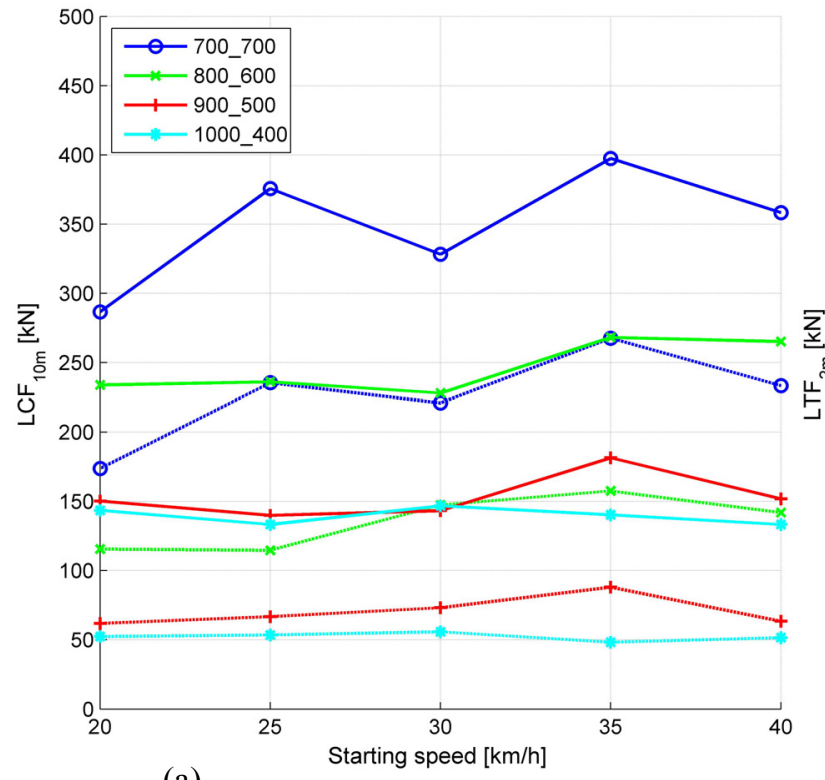

(a)

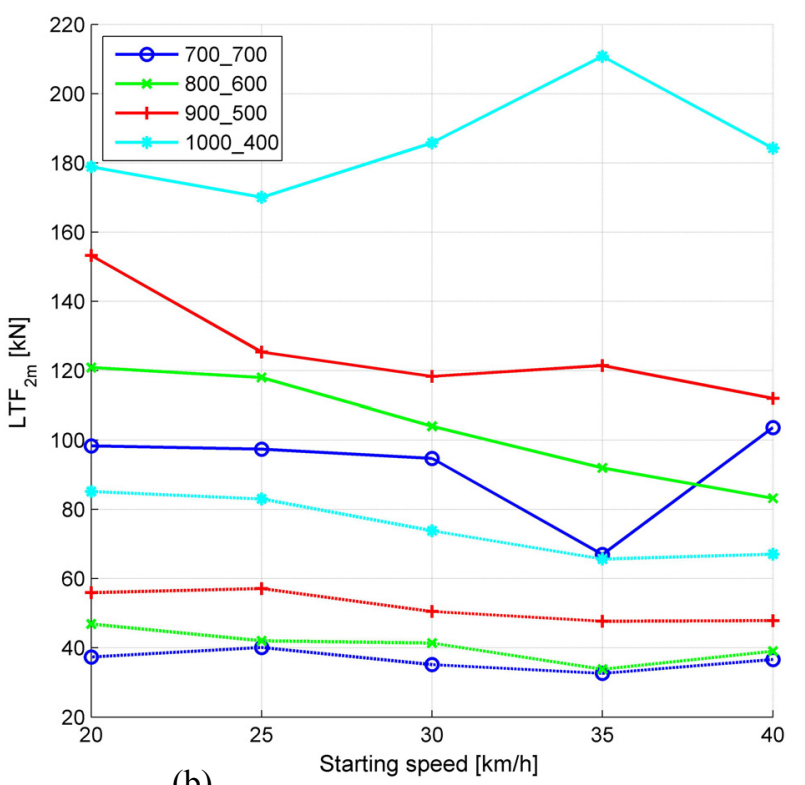

(b)

Figure 7: (a), dependency of $\mathrm{LCF}_{10 \mathrm{~m}}$ by starting speed, according to different position of remote locomotive (a); (b) similar to (a) except that it displays the $\mathrm{LTF}_{2 \mathrm{~m}}$. 


\section{Two remote locos}

This section deals with train sets equipped by two remote locos, which are assumed to have a synchronous behaviour (this case is not always verified, depending on the technology used for the communication of the locos). Two train sets concepts are investigated: coupled trains (i.e. three trains are put together sharing a section of their route) and fixed train composition, with one loco at the beginning another in the middle and last at the end of the train. Braking regimes used for coupled trains are LL (hauled mass is $1600 \mathrm{t}$ ) and G (with sub-train length of $500 \mathrm{~m}$, since in the next future $1500 \mathrm{~m}$ will be the more feasible maximum commercial length for long freight trains); braking regime used for fixed train composition is $\mathrm{G}$ (being $\mathrm{P}$ regime not feasible for such application, due to the high in-train forces).

Figure 8 shows cumulative function for previously described scenarios; it is clear the benefit of fixed compositions with respect to the coupled ones, even if tension forces are bigger than the counterparts. It is also important to emphasize here that fixed train compositions have a smaller dependence of in-train with respect to mass distribution compared to coupled trains.

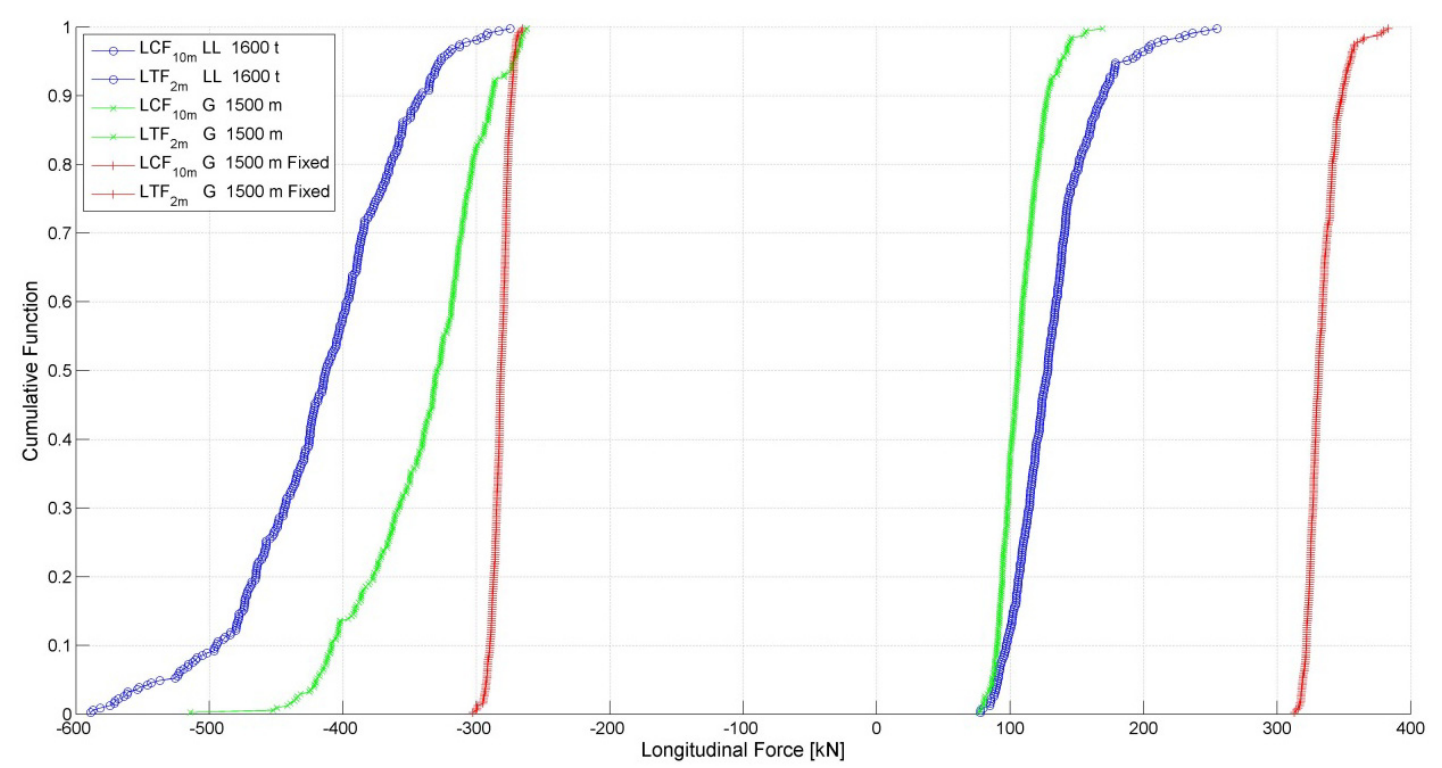

Figure 8: Cumulative function for train sets having two remote locos.

\subsection{Ad hoc loco activation}

Results previously shown have been obtained considering a synchronous braking of all locos; by means of an "ad hoc" choice of the activation sequence of remote locos, i.e. by changing the order with which brake pipe is vented, it is possible to change the values of in-train forces. From previous train sets, considering both connected trains and fixed compositions, the composition that had the higher sum of tensile and compressive forces has been selected, from each family, for the optimization by means of an ad hoc activation sequence of brake pipe venting. At this aim, two timings are defined: $\Delta \mathrm{T}_{1}$ and $\Delta \mathrm{T}_{2}$, the former indicates the activation 
delay of second loco with respect to the first, the latter indicates the activation delay of third loco with respect to the second; positive values mean a delay, negative values mean an anticipation. Figure 9 shows contour plots where the values are expressed in terms of percentages; such percentages are computed by dividing the maximum tensile force by the reference counterpart, doing the same with the compressive forces and then computing the mean of such values: $100 \%$ indicates that the combination of the two timings provides results similar to the reference case, higher values mean a worst condition (i.e. higher in-train forces), smaller values indicate a better condition. In this case, an ad hoc activation procedure is capable to provide only minor improvements with respect to the standard synchronous braking. Figure 9 also shows that optimized timings, for (a) connected trains and (b) fixed trains, are very different and a generalization is not possible for both families of train sets.

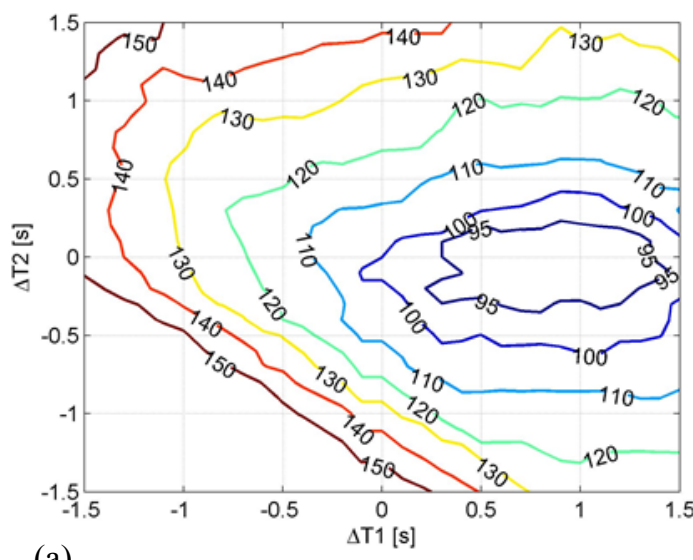

(a)

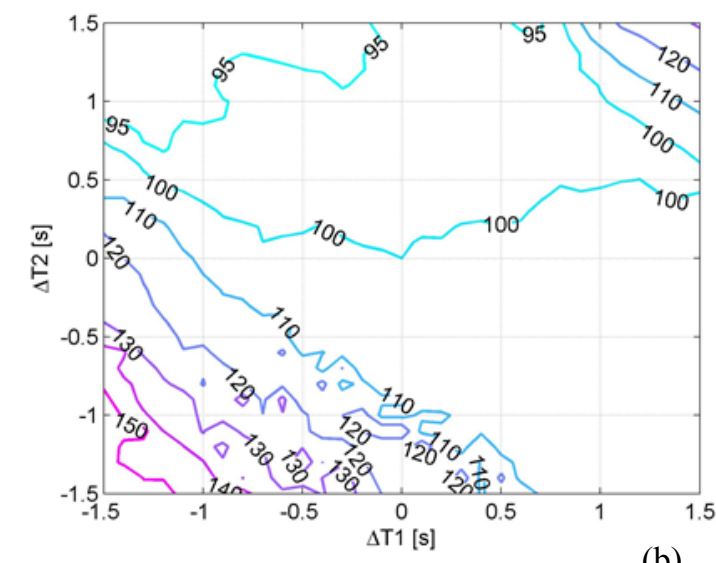

(b)

Figure 9: Contour plot for ad hoc activation of remote locos, (a) connected trains; (b) fixed composition.

\section{Conclusions}

The UIC certified TrainDy software has been here used for a first comparison of different braking technologies for future freight trains. The most promising technology is the electro-pneumatic brake applied on wagons equipped by disk brakes, hence by applying to freight trains the technologies already available for passenger trains. Another very promising technology, much more cost effective, is the employment of remote locos both for coupled train composition and for fixed train composition, being the latter more efficient with respect to in-train forces, but less manageable from the logistic point of view. An open issue for this type of technology is the communication strategy between the locos. For the train sets here considered, an ad hoc (smart) activation sequence of the drivers brake valves of remote locos does not provide significant benefits with respect to the synchronous activation, both for coupled train sets and for fixed train sets; on the contrary, the results here reported show the relevant benefits that can be obtained by an optimized distribution of the mass along the train for all the braking technologies here examined. 


\section{References}

[1] S. Iwnicki, Handbook of Railway Vehicle Dynamics, CRC Press, Boca Raton, 2006.

[2] L. Cantone, R. Karbstein, D. Negretti, L. Müller, "Validierung von TrainDy mit Versuchsergebnissen der Deutsche Bahn AG", ETR Eisenbahntechnische Rundschau, Nr. 5, pp. 234-239, 2009.

[3] L. Cantone, D. Negretti, A. Palazzolo, R. Karbstein, "Validazione dinamica di TrainDy con dati sperimentali DB e Trenitalia", Ingegneria Ferroviaria, Nr. 2, pp. 165-172, 2009.

[4] L. Cantone, T. Durand, "Longitudinal forces evaluation of SNCF trains", WCRR 2011, $9^{\text {th }}$ World Congress on Railway Research, Lille France 22-26 Maggio, 2011.

[5] L. Cantone, TrainDy: the new Union Internationale des Chemins de Fer software for freight train interoperability, Proc. IMechE, Part F: J. Rail and Rapid Transit, 2011, 225 (F1), 57-70. DOI 10.1243/09544097JRRT347.

[6] UIC 421 OR, "Rules of the consist and braking of international freight trains", $9^{\text {th }}$ edition, January 2012.

[7] L. Cantone, V. Vullo, "Analisi e ricerca di zone operative per treni merci a trazione distribuita", $42^{\circ}$ Convegno Nazionale Associazione Italiana per 1'Analisi delle Sollecitazioni (AIAS 2013), 11-14 Settembre 2013, Università degli Studi di Salerno.

[8] UIC 540 O, "Brakes - Air brakes for freight trains and passenger trains", $5^{\text {th }}$ edition, November 2006.

[9] L. Cantone, E. Crescentini, R. Verzicco, V. Vullo, "A numerical model for the analysis of unsteady train braking and releasing manoeuvres", Proc. IMechE, Part F: J. Rail and Rapid Transit, 2009, 223 (F3), 305-317. DOI 10.1243/09544097JRRT240.

[10] L. Cantone, D. Negretti, V. Vullo "Evaluation of the Admissible Longitudinal Compressive Forces by Means of Multibody Train Simulations", Proceedings of the First International Conference on Railway Technology: Research, Development and Maintenance, Las Palmas de Gran Canaria, Spain,18-20 April, 2012. 